\title{
Multi-Task Learning for Calorie Prediction on a Novel Large-Scale Recipe Dataset Enriched with Nutritional Information
}

\author{
Robin Ruede $^{1}$ Verena Heusser ${ }^{1} \quad$ Lukas Frank $^{1} \quad$ Alina Roitberg $^{2} \quad$ Monica Haurilet $^{2}$ Rainer Stiefelhagen ${ }^{2}$ \\ Institute for Anthropomatics and Robotics, Karlsruhe Institute of Technology, Germany \\ ${ }^{1}$ \{firstname. lastname\} @student.kit. edu, ${ }^{2}$ \{firstname. lastname\}@kit. edu
}

\begin{abstract}
A rapidly growing amount of content posted online, such as food recipes, opens doors to new exciting applications at the intersection of vision and language. In this work, we aim to estimate the calorie amount of a meal directly from an image by learning from recipes people have published on the Internet, thus skipping time-consuming manual data annotation. Since there are few large-scale publicly available datasets captured in unconstrained environments, we propose the pic $2 \mathrm{kcal}$ benchmark comprising 308000 images from over 70000 recipes including photographs, ingredients, and instructions. To obtain nutritional information of the ingredients and automatically determine the ground-truth calorie value, we match the items in the recipes with structured information from a food item database.

We evaluate various neural networks for regression of the calorie quantity and extend them with the multi-task paradigm. Our learning procedure combines the calorie estimation with prediction of proteins, carbohydrates, and fat amounts as well as a multi-label ingredient classification. Our experiments demonstrate clear benefits of multi-task learning for calorie estimation, surpassing the single-task calorie regression by $9.9 \%$. To encourage further research on this task, we make the code for generating the dataset and the models publicly available.

Index Terms-calorie estimation, multi-task learning, recipes, dataset, ingredients
\end{abstract}

\section{INTRODUCTION}

A question that many of us have asked ourselves is how healthy our diet is and what we should change for a more balanced lifestyle. Even though various diets and nutritional courses inform people how to live healthier, many still struggle to apply these rules in practice. Tracking the exact amount and quality (e.g., calories and macronutrient composition) of food eaten is important to successfully follow most diets. However, this can be particularly difficult, for instance when eating in a restaurant or canteen. Even when cooking ourselves, calculating these factors manually takes a lot of time and effort, leading to quick motivation loss and non-optimal results for many people. Guessing these values directly is also difficult as some studies show that most people are not able to correctly pinpoint the caloric value of a meal, mostly underestimating it [28].

In this work, we propose an approach for predicting the calorie content of one portion directly, in an end-to-end fashion from a single image of a meal. Since there is little work on calorie estimation and most of them only deal with images

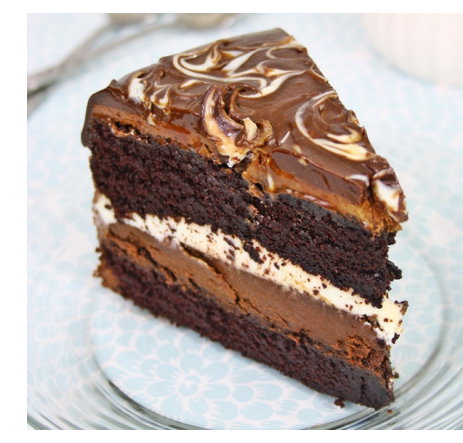

\begin{tabular}{lcr}
\multicolumn{3}{l}{ Nutrition Facts (per $100 \mathrm{~g})$} \\
\hline Calories & $\begin{array}{r}183 \\
\mathrm{kcal}\end{array}$ & $\begin{array}{r}198 \\
\mathrm{kcal}\end{array}$ \\
\hline Fat & $9 \mathrm{~g}$ & $9 \mathrm{~g}$ \\
\hline Carb & $17 \mathrm{~g}$ & $24 \mathrm{~g}$ \\
\hline Protein & $7 \mathrm{~g}$ & $4 \mathrm{~g}$ \\
\hline Ingredients (pred): Flour, \\
Butter, Milk
\end{tabular}

Fig. 1: Example image with accompanying nutritional values. The table shows the macronutrients and ingredients predicted by our model (pred) in comparison with the labels (true). Note that in this example we only consider the top-100 ingredients.

captured in constrained environments [26], we collect a largescale dataset of pictures of meals captured in the "wild". Our pic 2 kcal dataset comprises 308000 images of 70000 different recipes ranging from simple salads to cakes (fig. 1), pizzas, and soups. To collect our dataset and generate precise ground truth estimates, we propose a procedure based on the ingredients found in the recipe, instead of relying on user-given calorie estimates which are sparse and often incorrect. The ingredients and their mass given in the semi-structured section of the original recipe are each mapped to fully structured data such as calorie, macronutrient, and micronutrient amounts with the help of semantic embeddings created via free-text language models. These values are then aggregated to obtain the corresponding information for the finished dish.

We leverage our proposed dataset and implement different neural architectures for the calorie estimation task. To that end, we pose the calorie estimation task as a regression problem and evaluate convolutional neural networks (CNNs) previously used for image recognition by minimizing the L1 loss. We improve the performance of these conventional CNNs for image recognition and propose a network trained in a multi-task setting where additional nutritional information and ingredients are estimated. To foster further research in 
calorie estimation from unconstrained images, as well as for addressing other tasks concerning images of meals with structured recipe information, we make our code publicly available to the community ${ }^{1}$.

Overall, the contributions of this work are three-fold:

(1) We introduce a framework for retrieving nutritional information of recipes by matching ingredients and their mass to a nutrient database using phrase embeddings.

(2) We build a large-scale recipe dataset consisting of naturally collected dish images and their recipes collected from a German cooking website.

(3) We propose a multi-task end-to-end approach for calorie and nutrient estimation based on a single image of the dish.

\section{RELATED WORK}

Calorie Estimation. In existing proprietary products, calorie tracking is mostly carried out manually by looking up specific ingredients and amounts for each eaten meal. There exist a large number of automated dietary tracking approaches using mobile applications such as [14]. However, these require a lot of user intervention and a high amount of manual input. For instance, the users are required to specify portion sizes and sometimes even ingredients manually, making this approach time-intensive and error prone.

In this work, we address the task of vision-based food analysis, which aims to predict nutritional information based on images of the dish. The majority of vision-based works for food analysis (overview of methods and datasets in [26]) consist of a multi-stage procedure [8], [20], [23], [29]. In these approaches, the image is segmented pixel-wise into food- and non-food components, which is followed by the classification of the image into a fixed set of categories, such as cuisine or food type. The next stage consists of volume and weight estimation, and a nutrient information prediction step. The nutritional information is predicted based on extracted features from pre-trained $\mathrm{CNNs}$ restricted by the segments generated in the first stage. For example, Myers et al. [20] segmented a given food image, generated the food categories, the ingredients, and the corresponding volume. The calories were then predicted by matching the estimated volume and the calorie density of the food category against a nutritional database. Moreover, meta-data such as GPS location and user's food preferences were used to improve the predictions.

Although multi-stage approaches improve several baseline methods for calorie estimation they have a number of disadvantages. First, these methods require the definition and separate optimization at each stage, where each separate subtask in the different phases is difficult to solve accurately [19], [26]. Moreover, these models comprise a segmentation step for classifying the pixels into food and non-food categories and the estimation of the volume of the meal. Thus, one would require pixel-wise annotations of a high amount of data as well as additional information specific to each meal in the image such

${ }^{1}$ Code available at https://github.com/phiresky/pic2kcal/ as its volume. Furthermore, multi-stage approaches require a definition of the different stages as well as the inputs and outputs for every stage, respectively. This entails that potentially useful information from the earlier stages is not passed on and can not be used by the later stages to improve the predictions.

End-to-end Approaches for Food Related Tasks. End-toend approaches rely on substituting the pipeline of multi-stage approaches by a single model. Instead of training a pipeline of models addressing different subtasks, a single network is applied on the input data and directly estimates the desired final output. Thus, only the raw inputs and final outputs (next to the end-to-end architecture itself) need to be specified, while the neural network can learn which information is relevant internally. End-to-end models do not struggle with the problems of multi-stage approaches, and have therefore replaced multi-stage approaches in a number of computer vision domains. While end-to-end methods have shown great promise in tasks such as image recognition [11], there are currently few end-to-end models for food related tasks such as calorie or nutrient prediction [6], [9], [25].

Multi-task Approaches. Multi-task learning [4] is a method for addressing several different tasks concurrently with the overall aim of improving generalization by exploiting commonalities between separate problems. [6], [9] show that the performance of food related tasks such as ingredient prediction can be improved through multi-task learning. Takumi et al. [9] proposed a multi-task $\mathrm{CNN}$ which estimates the calories together with the average ingredient word embedding, the type of food, and an embedding of the preparation steps of the meal. [25] introduced a multi-task CNN to jointly predict the food category, calories, and the salinity given a food image which was evaluated on an unpublished dataset.

Recipe Datasets. Several works use variations of the Food101 [3] dataset to address food image recognition tasks (e.g., [10], [20], [27]). The Food-101 dataset consists of altogether $101 \mathrm{k}$ pictures of dishes sorted into 101 categories. Takumi et al. [9] collected a dataset of English and Japanese recipes including ingredients and user-given calorie estimates that was not made publicly available. Most other datasets consist of images captured in constrained environments, such as a specific canteen [1] or contain images of single food items instead of whole dishes [16]. Furthermore, many datasets contain cuisine-specific data, such as images of just Chinese dishes [6] or American dishes [3], [7]. Hence, there is a lack of generic datasets containing various dishes from around the globe and recorded under unconstrained circumstances, i.e. captured in the wild [26]. While most datasets contain food category and ingredient labels, there are few datasets containing nutritional and calorie annotations.

The largest currently available dataset is Recipe1M [17], which encompasses one million images extracted from websites comprising categories, ingredients, and instructions. The authors also expanded upon the dataset by adding nutritional information and pictures sourced from web searches using 
the recipe title (Recipe $1 \mathrm{M}+)$. Although this greatly increases the size of the dataset, the images are also far less relevant to the specific recipes and include images of similar dishes, prepackaged goods, and images taken by professional photographs. The nutritional information was extracted using the USDA Food Database [21] by matching ingredient quantities and names semi-manually. We compare our collected pic $2 \mathrm{kcal}$ dataset to Recipe1M+ without web-search sourced pictures. Within Recipe $1 \mathrm{M}+$, around $20 \mathrm{k}$ recipes with images have nutritional information, which totals to $50 \mathrm{k}$ images.

\section{DATASET}

\section{A. Data Collection}

We collect our dataset from a popular recipe website that contains ingredient lists, cooking instructions, and pictures of the resulting meals. The recipes are from a large number of different cuisines, ranging from baked dishes to drinks, snacks, and dietary meals. Most recipes have at least one picture, which are either uploaded by the original author of the recipe or by third parties following the instructions in the recipe. Since the images are diverse and can comprise a single plate of food, while other consist of the entire dish, such as an entire casserole, it is not possible to directly obtain the information if an image contains a single portion or more. Around $10 \%$ of recipes include a user-given value for the amount of calories in each portion that the recipe supposedly has. We choose to use a German recipe website over American websites as a data source as American sites typically denote the required amount of a specific ingredient in terms of its volume, i.e., in number of cups or teaspoons, which are a more inaccurate measure with high variance in the actual mass. Contrary, in German recipes, the amount of an item is mostly given by its mass, i.e. in grams. Our resulting dataset also covers additional metadata such as the type of meal (e.g., dessert or side dish), the average user rating, the preparation time, and additional properties.

\section{B. Preprocessing}

The dataset only has user-given calorie information for a small part of the data and does not include any details regarding the macronutrient composition. Furthermore, since the information provided by the user is often inaccurate, we match the list of ingredients against a database of nutritional values to sum up the proportions of macronutrients as well as the total calories. To facilitate this, we collect a secondary dataset from a German website of nutritional values, which contains values for the amount of fat, protein, and carbohydrates in grams per 100 grams of product. The data is partially sourced from the USDA Food Composition Database [21], and partially crowd sourced from manufacturer-given data of specific products. Additionally, it contains food quantities such as "1 mediumsized apple $=130 \mathrm{~g}$ ", which we used to convert human-intuitive amounts that are commonly used in recipes (like add " 1 piece" of fruit) to their equivalent mass. Thus, the processing of the recipes is comprised of two stages:
(1) Matching the ingredients to the nutritional database. For example, "medium-size white or Yukon Gold potatoes, peeled, cut into 1-inch cubes" becomes potatoes.

(2) Calculating the amount of different ingredients in the recipe. For example, "13/4 cups diced potatoes" is mapped to 394 grams in the second step of our proposed processing pipeline.

Matching Ingredients. Since the ingredients are user-given and can be noisy, the mapping of the recipe ingredients to the nutritional database is not straightforward. The given ingredient name often includes information that is not relevant to the product itself, but rather to its preparation or visual qualities. These additional text snippets are hard to separate from information that is relevant, e.g., "3 onions, diced" and "3 onions, in slices" refer to the same product, while " $500 \mathrm{~g}$ pasta, cooked" and "500g pasta, raw" vary significantly in their calorie density. Thus, since matching the textual representation of the ingredient from a recipe to the ingredient from our nutritional database is ambiguous, we evaluate different matching methods on the text content of our dataset.

First, a simple option is to directly match the text of each ingredient to the nearest ingredient based on character edit distance. However, this results in low quality matchings due to missing handling of synonyms and the above mentioned issue of irrelevant information in the description of the ingredient. To address this, we tokenize the ingredient name to words and embed each word to a vector with Word2Vec [18] or FastText [2]. This is then averaged over the word vectors to get an ingredient embedding, in the same way as in the FastText library for extracting sentence vectors. This still leads to unsatisfactory results, since each word in the ingredient name has the same weight, even though some words specify less important details. For example in "red onion" vs "red apple", the word "red" is much less important than "onion" and "apple". Finally, we obtain the best result by using the Google Universal Sentence Encoder [5], which can create 512-dimensional embeddings for an arbitrary amount of input text. We find the best matches for an ingredient by first comparing the embedding of the user-given free text from the recipe to the embeddings for all food items for which we have nutritional data using the cosine similarity (in our case this is equivalent to the dot product, since the embedded vectors are all normalized).

Ingredient Amount Calculation. The second problem we have to tackle for pre-processing the dataset is calculating the normalized gram or milliliter amounts of ingredients in the recipes. For ingredients given in grams, this is trivial, but for many items the recipe authors use other units of measure, e.g., can, piece, tablespoon, some, or salt "by taste". Since spices usually have little impact on the nutritional values, we exclude ingredients that are given "by taste" and similar. For the other amounts, we match the unit name (like "tablespoon" or "medium large") exactly and multiply it with the given amount. We also add some special cases like matching "can" to "can (drained weight)" and similar. This amount 
matching is applied to all possible ingredients that have a cosine similarity of more than 0.84 in descending order until a matching amount is found. If the amount matching fails, the ingredient is marked as unmatched, and if a recipe has at least one unmatched ingredient, it is completely discarded in our dataset. We immediately discard recipes without perfect matches to be sure our dataset is accurate at the cost of losing a number of mostly good samples. As a final step, we filter out all data points where the summed up calories of the recipe is outside of two standard deviations from the mean of all recipes. We perform this step iteratively, until the outliers removal converges (i.e., no more outliers are found). This is necessary because some recipes contain obviously wrong information (for example, in one carrot cake recipe the author specified to use a million carrots).

\section{Dataset Statistics}

Overview. We found $211 \mathrm{k}$ recipes that contain at least a single image of the underlying recipe, of which around 20k recipes have user-given calorie information, which we, however, do not use for training our models. In total, we collected 900k pictures where on average each recipe has four images, while the recipes comprise a total of $374 \mathrm{k}$ unique ingredients. This high number is caused by slight differences in spelling or irrelevant details. The database of nutritional values contains a total of 390k ingredients, which we filter by duplicates and popularity to $123 \mathrm{k}$ ingredients before matching the ingredients to the recipes. After matching the ingredients found in the recipes with the food database, the count reduces to 5700 unique ingredients.

Table I shows statistics for each of the matching and preprocessing steps described in section III-B. The final recipe count varies depending on whether we aggregate calories per recipe, per portion or per $100 \mathrm{~g}$ of raw mass. We discard around $60 \%$ of recipes during matching as we remove all recipes that have ingredients which do not fully match. This is intentional as we want to ensure that we only retain data points that are accurate, and the proportion of usably matched recipes could be improved with further tweaking. When aggregating per portion, we exclude more data points since we have to discard all recipes where the user did not supply information on how many portions a recipe contains.

Moreover, we show the amount of recipes discarded for each of our three proposed preprocessing steps. First, 127k recipes contain an ingredient list that we were not able to completely match with the food database. Even though imperfect matching of the ingredients and discounting unmatched ingredients would still lead to good estimates, we discard these cases to make sure that we do not worsen our approximation.

Finally, a total of $11 \mathrm{k}$ recipes do not contain any information per portion, so we cannot estimate the calorie values in these cases. We experience strong outliers that are clearly incorrect in less than $21 \mathrm{k}$ recipes, which we discard in the final stage of our preprocessing pipeline.
TABLE I: Preprocessing steps (each described in section III-B) and corresponding sample counts depending on the amount of food for that the calories are estimated.

\begin{tabular}{lccc}
\hline Preprocessing & Per portion & Per $100 \mathrm{~g}$ & Per recipe \\
\hline Original recipes count & $211 \mathrm{k}$ & $211 \mathrm{k}$ & $211 \mathrm{k}$ \\
$\begin{array}{l}\text { Removed (incomplete } \\
\text { ingredients match) }\end{array}$ & $127 \mathrm{k}$ & $127 \mathrm{k}$ & $127 \mathrm{k}$ \\
$\begin{array}{l}\text { Removed (no portion } \\
\text { size information) }\end{array}$ & $31 \mathrm{k}$ & 0 & 0 \\
Removed (kcal outliers) & $11 \mathrm{k}$ & $14 \mathrm{k}$ & $21 \mathrm{k}$ \\
\hline Final recipe count & $\mathbf{4 2 k}$ & $\mathbf{7 0 k}$ & $\mathbf{6 3 k}$ \\
\hline
\end{tabular}

TABLE II: Comparison of sample and recipe counts of Recipe1M+ [17] with our dataset. Recipe1M+ does not include portion information. Note that due to the parsing errors described in section III-C a large fraction of Recipe1M+ recipes included in these counts is not usable for training models relying on the ingredient amounts (such as calorie prediction).

\begin{tabular}{llccc}
\hline Dataset & Property & Per portion & Per 100g & Per recipe \\
\hline \multirow{4}{*}{ Recipe1M+ } & Mean [kcal] & N/A & 219 & 1047 \\
& Std. Dev. [kcal] & N/A & 129 & 658 \\
& Recipe count & N/A & $17 \mathrm{k}$ & $10 \mathrm{k}$ \\
& Sample count & N/A & $\mathbf{4 4 k}$ & $\mathbf{2 4 k}$ \\
\hline \multirow{5}{*}{ pic2kcal } & Mean [kcal] & 425 & 179 & 1791 \\
& Std. Dev. [kcal] & 207 & 73 & 1007 \\
& Recipe count & $42 \mathrm{k}$ & $70 \mathrm{k}$ & $63 \mathrm{k}$ \\
& Sample count & $\mathbf{1 7 9 k}$ & $\mathbf{3 0 8 k}$ & $\mathbf{2 6 7 k}$ \\
\hline
\end{tabular}

Dataset Comparison. In total, our dataset contains $308 \mathrm{k}$ images with associated calorie estimate, as each of the $70 \mathrm{k}$ recipes includes on average multiple pictures. Table II shows a comparison between our pic $2 \mathrm{kcal}$ dataset and the Recipe1M+ dataset. When excluding web-search sourced images, the Recipe1M+ dataset has around 20k recipes with images and nutritional information. After applying the same outlier filtering to Recipe1M as for our own dataset, Recipe1M+ remains with $17 \mathrm{k}$ recipes with $44 \mathrm{k}$ images. When aggregating per recipe, Recipe1M+ loses half the recipes (around 10k) during the outlier filtering. The reason for this high number of excluded recipes is that the dataset includes a large amount of incorrectly parsed fractions (see [15, sec. 4.1]), which causes the ingredient amounts and thus the total mass to diverge by orders of magnitude in around $64 \%{ }^{2}$ of the parsed recipes (for example, $1 / 4$ cup is mapped to 14 cups).

Data Split. We split the recipes into train, validation, and test set $(70 \%, 15 \%$, and $15 \%$, respectively), where we made sure that pictures of the same recipe are in the same data split.

Ingredient Distribution. Table III shows the frequency of the 15 most frequent ingredients in our dataset. As can be seen, spices (e.g., salt, pepper) and popular baking components (e.g., flour) are the strongest represented ingredients in our data.

\footnotetext{
${ }^{2}$ We manually compared parsed ingredients of 100 randomly selected recipes of the Recipe $1 \mathrm{M}+$ dataset to their original on the websites.
} 


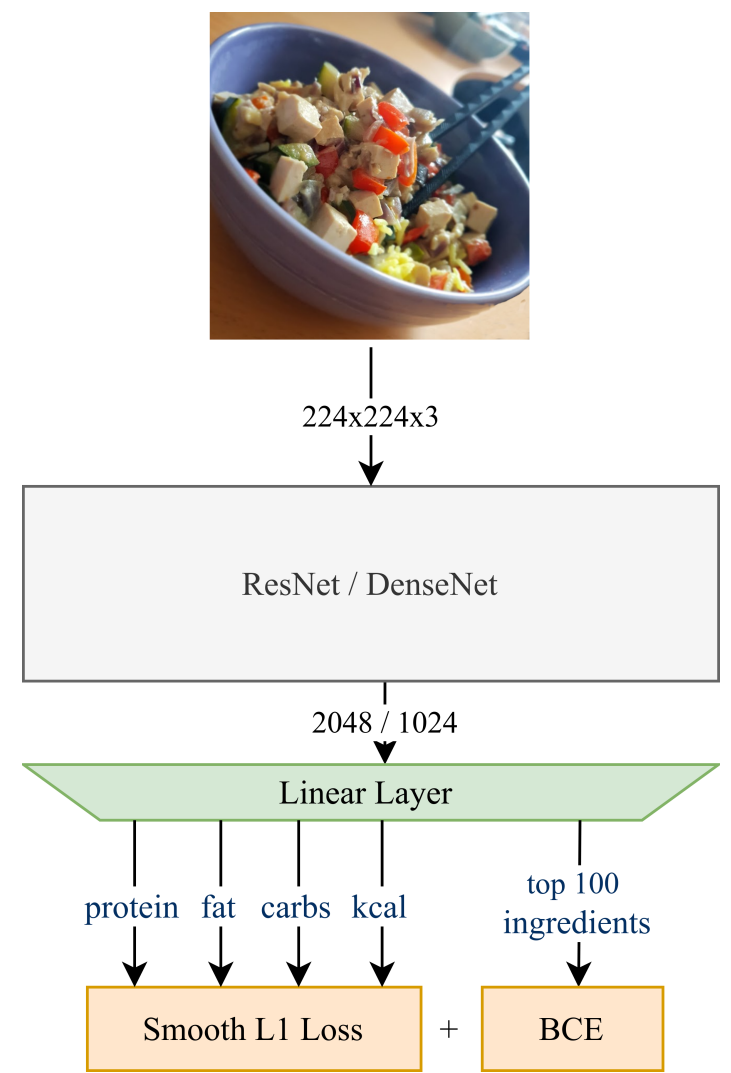

Fig. 2: Overview of our proposed architecture. Our model is trained in a multi-task setup inferring the calories, the ingredients, and the macronutrients (e.g. protein).

\section{METHODS}

In this section, we describe the architectures we adopt for calorie estimation as well as our enhancement of these models with the multi-task paradigm. We use end-to-end algorithms, i.e. the networks operate on the raw food image without intermediate processing steps. Similar to other multi-task approaches in food image processing [6], [9], [25], we leverage pre-trained networks that are popular in the computer vision community. For this, we adopt multiple variants of the prominent ResNet [11] and DenseNet [13] architectures as our backbone models, which were pre-trained on ImageNet [24]. As the original models were developed for classification, while we aim for calorie regression, we replace the last fully-connected layer with a regression layer and keep the rest of the architecture unchanged.

We argue that ground truth values of macronutrients correlate with the calorie amount and carry useful information for calorie estimation. We therefore propose to include this additional knowledge as a supplemental learning signal to the calorie estimation network by following a multi-task learning paradigm. Our extension modifies the last fully connected layer of the network and does not alter the core architecture. Originally, the models predict the calorie value (in kcal) by using a single output neuron. We enhance this architecture with three additional regression neurons for protein, fat, and
TABLE III: Most common ingredients after matching.

\begin{tabular}{lc}
\hline Ingredient & Number of recipes \\
\hline Salt & 119244 \\
Sugar & 59066 \\
Chicken Egg & 58185 \\
Flour & 46069 \\
Butter & 45891 \\
Onion, fresh & 41206 \\
Milk & 24531 \\
Vanilla Sugar & 24011 \\
Oil & 22822 \\
Paprika & 22781 \\
Garlic & 21348 \\
Water & 20359 \\
Pepper & 19336 \\
Olive Oil & 18928 \\
Baking Powder & 15966 \\
Cream & 15039 \\
\hline
\end{tabular}

carbohydrates (in grams) in a multi-task network.

Our multi-task model regresses the macronutrient information with additional binary (sigmoid) outputs to predict the top $n=100$ ingredients. The resulting final layer therefore has four regression outputs for the calories and macronutrients and $n$ binary outputs for the top $n$ ingredients (as we show in fig. 2). We then compute the final loss by combining the smooth L1 loss for the regression outputs and the Binary Cross Entropy (BCE) loss for the top-100 ingredients:

$$
\text { multi-task loss }=\mathrm{L} 1_{\text {kcal }}+\sum_{m \in\{\text { fat,prot,carb }\}} \mathrm{L} 1_{m}+\gamma \cdot \mathrm{BCE}
$$

To get the same scaling of the learning signals, we multiply the BCE loss with a factor $\gamma$ depending on the dataset, therefore balancing the losses to have roughly equal contribution.

As there is no related work on published datasets targeting the same task with similar data, and no common baseline used in related work, we implement a baseline predicting the mean of all training samples for comparison. This is a simple method that still includes the general statistical distribution of the dataset. We also add an alternative baseline using the values from a random recipe in the dataset as the prediction. Since hitting the correct recipe is very unlikely, this baseline is equivalent to predicting a random gaussian value with the mean and variance of the dataset, which has worse performance than always predicting the mean value. We use the same baselines for the macronutrient regression.

\section{Evaluation}

\section{A. Setup}

To evaluate the model performance and create a competitive benchmark, we conduct extensive experiments with multiple neural networks (various ResNet [11] and DenseNet [13] variants). All models are implemented using PyTorch [22] and are trained for 25 epochs using a batch size of 50 .

Our initial goal was to predict the calories of a meal shown in an image. Since the dataset does not provide information for how much food is in one specific image - and images can 
contain both a single served plate of food as well as a full pot - we run the following types of experiments:

- calorie prediction per recipe

- calorie prediction per portion

- calorie prediction per $100 \mathrm{~g}$ of raw ingredient mass

Our first set of experiments leverages raw unfiltered data and results in an insufficient performance of the models. Recognition results were further improved by adding the quality and outlier filtering described in section III. We also evaluated a model in a classification setup instead of using the regression losses, however, the performance did not improve.

Furthermore, we run experiments to analyze the impact of additional information on the performance of our neural architectures. Within these experiments, we leverage the following three levels of additional information:

- kcal-only: As a reference, we predict the calories directly with no further information.

- kcal + nutritional information: In addition to calories, the model predicts protein, fat, and carbohydrate amounts, which allows it to learn explicitly about the different components adding up to the food energy of a meal.

- kcal + nutritional information + top-100 ingredients: The model also predicts which of the top-100 most common ingredients of the dataset are present in the dish. This further supports it in predicting the calories since it can more easily learn the correlation between different ingredients and the macronutrients.

\section{B. Results}

To evaluate the quality of calorie prediction, we adopt the relative error (rel_error $=1-\left|\frac{\text { pred }}{\text { truth }}\right|$ ) as our main metric. In addition, we provide the absolute error (L1 error) of calories (in kcal), fat, protein, and carbohydrates (each in grams). In this setting, using the relative error is not very helpful, since in many dishes there is at least one absent macronutrient value. In tables IV,V, and VI, the first column depicts the relative and the other columns show the absolute error (either in kcal or grams). We compute the baselines as described in section IV and subsequently compare the results for three different tasks.

In table IV, we show the results of our baseline methods for the macronutrient and calorie estimation. As expected, the random baseline has a poorer performance than the mean estimate having a discrepancy in absolute error of around 23. Then, we compare different backbone architectures for our multi-task setup. As we see, the two DenseNet [13] versions yield the highest performance (a relative error of 0.326 and absolute error of $46.9 \mathrm{kcal}$ for calorie estimation in case of DenseNet121). In contrast, the ResNet and ResNeXt [12] architectures show an error of more than 0.33 .

Next, we compare calorie regression for different amounts of food (table V), as the actual food quantity present in the photograph is not explicitly annotated in our dataset, and is only implicitly inferred via the portion- and recipe sizes. The best performance is reached when predicting the amount of calories per $100 \mathrm{~g}$. This can be attributed to the following:
TABLE IV: Relative and absolute regression error (in kcal / grams) by model architecture, each predicting calories, macronutrients, and ingredients per $100 \mathrm{~g}$ in a multi-task setting. In this setup, DenseNet achieves the best performance.

\begin{tabular}{lccccc}
\hline Model & kcal (rel) & kcal & protein & fat & carbs \\
\hline Rand. Baseline & 0.595 & 83.3 & 4.36 & 6.32 & 15.0 \\
Mean Baseline & 0.464 & 60.5 & 3.10 & 4.49 & 10.5 \\
\hline ResNet50 & 0.334 & 47.8 & 2.54 & 3.93 & 7.13 \\
ResNet101 & 0.336 & 48.2 & 2.54 & 3.94 & 7.17 \\
ResNext50_32×4d & 0.33 & 47.2 & 2.50 & 3.89 & 6.99 \\
DenseNet121 & $\mathbf{0 . 3 2 6}$ & 46.9 & 2.51 & 3.88 & 6.97 \\
DenseNet201 & 0.327 & 47.2 & 2.53 & 3.89 & 7.04 \\
\hline
\end{tabular}

TABLE V: Relative and absolute regression error (in kcal / grams) depending on the amount of food for which the nutritional values were predicted. Each model is predicting calories, macronutrients, and ingredients using a multi-task DenseNet121 backbone model.

\begin{tabular}{lcccccc}
\hline & amount & kcal (rel) & kcal & protein & fat & carbs \\
\hline Rand. BL & \multirow{2}{*}{ portion } & 0.909 & 235 & 15.5 & 16.0 & 30.8 \\
Mean BL & 0.736 & 170 & 11.2 & 11.4 & 22.2 \\
Ours & & $\mathbf{0 . 6 2 3}$ & 154 & 9.21 & 10.7 & 19.1 \\
\hline Rand. BL & \multirow{2}{*}{ recipe } & 1.37 & 1130 & 57.7 & 73.0 & 167 \\
Mean BL & 1.23 & 858 & 41.9 & 54.4 & 125 \\
Ours & & $\mathbf{0 . 8 2 3}$ & 711 & 34.8 & 46.9 & 94.4 \\
\hline Rand. BL & \multirow{2}{*}{$100 \mathrm{~g}$} & 0.595 & 83.3 & 4.36 & 6.32 & 15.0 \\
Mean BL & 0.464 & 60.5 & 3.10 & 4.49 & 10.5 \\
Ours & & $\mathbf{0 . 3 2 6}$ & 46.9 & 2.51 & 3.88 & 6.97 \\
\hline
\end{tabular}

TABLE VI: Relative and absolute regression error (in kcal / grams) by prediction task, each predicting values per $100 \mathrm{~g}$ using a DenseNet121 backbone. The multi-task model that predicts the calories, macronutrients, and top-100 ingredients simultaneously has the best performance for all properties.

\begin{tabular}{lccccc}
\hline & kcal (rel) & kcal & protein & fat & carbs \\
\hline Random Baseline & 0.595 & 83.3 & 4.36 & 6.32 & 15.0 \\
Mean Baseline & 0.464 & 60.5 & 3.10 & 4.49 & 10.5 \\
Kcal-only & 0.362 & 50.3 & N/A & N/A & N/A \\
$\dddot{+}$ macros & 0.345 & 49.0 & 2.67 & 4.06 & 7.70 \\
¥̈+ top-100 ingredients & $\mathbf{0 . 3 2 6}$ & 46.9 & 2.51 & 3.88 & 6.97 \\
\hline
\end{tabular}

(1) we collected the largest amount of data samples for the $100 \mathrm{~g}$ setting. (2) the size of a portion and of a recipe can be very subjective and noisy, while $100 \mathrm{~g}$ is a more exact measurement. Note that this remark also holds when using the baseline models (table V).

Finally, we examine the effect of the multi-task learning paradigm which we propose in section IV, i.e. simultaneously predicting the macronutrient amounts (fat, carbohydrates, protein) and the presence of ingredients in addition to the calorie amount (table VI). Multi-task learning clearly elevates the performance of our model in all prediction tasks. For 


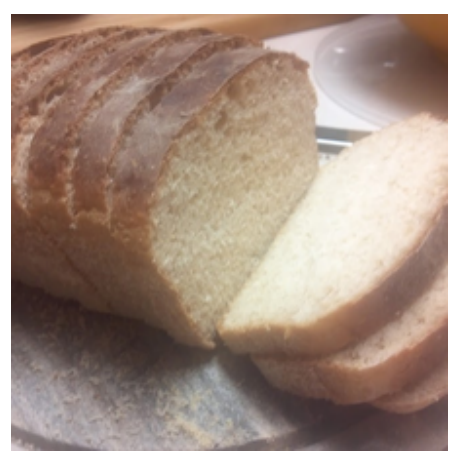

Nutrition Facts (per $100 \mathrm{~g}$ )

\begin{tabular}{lrr}
\hline & Pred & True \\
\hline Calories & $\begin{array}{r}229 \\
\mathrm{kcal}\end{array}$ & $\begin{array}{r}239 \\
\mathrm{kcal}\end{array}$ \\
\hline Fat & $3 \mathrm{~g}$ & $2 \mathrm{~g}$ \\
\hline Carb & $44 \mathrm{~g}$ & $46 \mathrm{~g}$ \\
\hline Protein & $7 \mathrm{~g}$ & $7 \mathrm{~g}$ \\
\hline Ingredients (pred): Flour \\
\hline Ingredients (true): Oil, \\
Flour \\
\hline
\end{tabular}

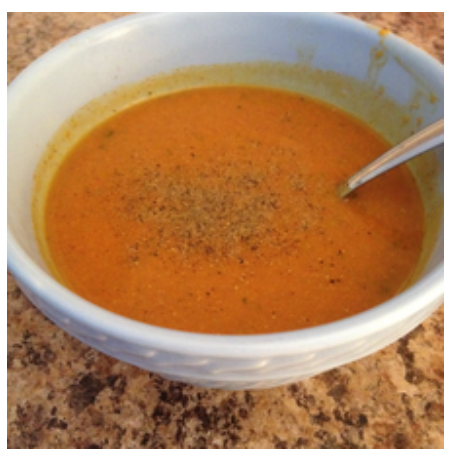

\begin{tabular}{lrr}
\hline \multicolumn{3}{l}{ Nutrition Facts (per 100 g) } \\
\hline & Pred & True \\
\hline Calories & $\begin{array}{r}99 \\
\mathrm{kcal}\end{array}$ & $\begin{array}{r}59 \\
\mathrm{kcal}\end{array}$ \\
\hline Fat & $8 \mathrm{~g}$ & $4 \mathrm{~g}$ \\
\hline Carb & $7 \mathrm{~g}$ & $5 \mathrm{~g}$ \\
\hline Protein & $3 \mathrm{~g}$ & $1 \mathrm{~g}$ \\
\hline Ingredients (pred): & \\
\hline & Ingredients (true): Garlic
\end{tabular}

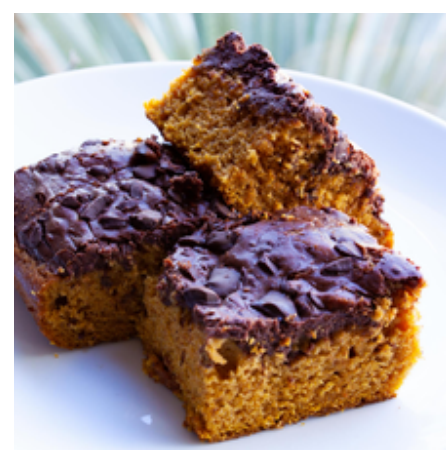

Nutrition Facts (per $100 \mathrm{~g}$ )

\begin{tabular}{|c|c|c|}
\hline & Pred & $\overline{\text { True }}$ \\
\hline Calories & $\begin{array}{r}252 \\
\text { kcal }\end{array}$ & $\begin{array}{r}241 \\
\text { kcal }\end{array}$ \\
\hline Fat & $12 \mathrm{~g}$ & $9 \mathrm{~g}$ \\
\hline Carb & $28 \mathrm{~g}$ & $33 \mathrm{~g}$ \\
\hline Protein & $8 \mathrm{~g}$ & $6 \mathrm{~g}$ \\
\hline
\end{tabular}

Ingredients (true): Egg, Flour, Baking powder, Cacao powder

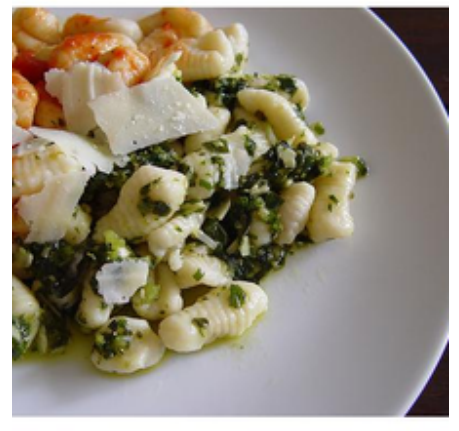

\begin{tabular}{lrr}
\hline \multicolumn{3}{l}{ Nutrition Facts (per $100 \mathrm{~g})$} \\
\hline & Pred & True \\
\hline Calories & $\begin{array}{r}190 \\
\mathrm{kcal}\end{array}$ & $\begin{array}{r}229 \\
\mathrm{kca}\end{array}$ \\
\hline Fat & $9 \mathrm{~g}$ & $17 \mathrm{~g}$ \\
\hline Carb & $20 \mathrm{~g}$ & $13 \mathrm{~g}$ \\
\hline Protein & $6 \mathrm{~g}$ & $4 \mathrm{~g}$ \\
\hline Ingredients (pred): & \\
\hline Ingredients (true): Onions, \\
Garlic, Parsley
\end{tabular}

Fig. 3: Example predictions of our multi-task model for different types of dishes. We report the predicted calories, fat, protein, carbohydrates, and ingredients from the validation set. We show that the predictions are close to the ground truth, in particular the model can easily distinguish high- and low-calorie foods (e.g., between bread and soup). example, our multi-task model decreases the relative error by $9.9 \%$, compared to the calorie-only approach. This shows that the model learns the interplay between the amount of different macronutrients and the calorie quantity, as well as between the ingredients and the calories, even though how these are correlated (e.g. $1 \mathrm{~g}$ of protein $=4 \mathrm{kcal}$ ) is not explicitly defined during training.

In fig. 3 we provide multiple examples of our model predictions for different food photographs and the corresponding ground truth. While our model does not give perfect calorie predictions, it discriminates well between high- and low-calorie dishes. For example, the soup image is assigned 99 calories, contrary to a chocolate cake with a prediction of 252 calories (the ground truth values are 59 and $241 \mathrm{kcal}$, respectively). In the examples, the model shows a better performance at predicting calories for baked goods, but has problems with dishes that have a greater variety of components, such as soups, where our model has not inferred any of the top-100 ingredients. This trend is reflected in the results on the whole dataset, which is to be expected as baked dishes often require similar ingredients such as flour or eggs.

\section{CONCLUSION AND Future WORK}

We address the problem of end-to-end calorie estimation from food images. To that end, we propose a collection scheme for generating ground truth calorie estimates by matching the recipes to a food database. We employ our collection scheme and create the large-scale pic $2 \mathrm{kcal}$ dataset totaling 70000 recipes with 308000 associated images with ingredients and nutritional information. We use the collected dataset to evaluate baselines and several neural networks previously designed for image classification. We show that performance is improved through the use of multi-task learning, where the model simultaneously predicts the ingredients of the meal and macronutrients in addition to the calorie estimation.

In this work, even though we focus on calorie estimation, the dataset contains additional attributes that can characterize the meals. In addition to calories, we include the type of the meal (such as dessert or side dish), ingredient quantities, cooking instructions, an average user rating, preparation time, tags, and further properties. Thus, a possible future work would be to use these additional properties to further improve the calorie prediction models in a similar manner to our multi-task setup where we leverage the macronutrients and ingredients.

The quality of the ingredient matching (III-B) could be improved by fine-tuning the sentence embedding model used to match the free-form ingredient names by using the cooking instructions as a text corpus. Further problems related to food computing could also be approached using the pic $2 \mathrm{kcal}$ dataset, like detecting if a meal is vegan or fits a specific diet (low-carb, paleo, keto, etc.). Finally, we hope that the pic2kcal dataset paves the way for mobile nutrition applications as the data is collected in the wild, and most images are essentially captured with smartphone cameras. 


\section{REFERENCES}

[1] Oscar Beijbom, Neel Joshi, Dan Morris, Scott Saponas, and Siddharth Khullar. Menu-match: Restaurant-specific food logging from images. In 2015 IEEE Winter Conference on Applications of Computer Vision, pages 844-851. IEEE, 2015.

[2] Piotr Bojanowski, Edouard Grave, Armand Joulin, and Tomas Mikolov. Enriching Word Vectors with Subword Information. Transactions of the Association for Computational Linguistics, 5:135-146, December 2017.

[3] Lukas Bossard, Matthieu Guillaumin, and Luc Van Gool. Food-101 mining discriminative components with random forests. In European Conference on Computer Vision, 2014.

[4] Rich Caruana. Multitask learning. Machine learning, 28(1):41-75, 1997.

[5] Daniel Cer, Yinfei Yang, Sheng-yi Kong, Nan Hua, Nicole Lyn Untalan Limtiaco, Rhomni St John, Noah Constant, Mario Guajardo-Céspedes, Steve Yuan, Chris Tar, Yun-hsuan Sung, Brian Strope, and Ray Kurzweil Universal Sentence Encoder. In In submission to: EMNLP demonstration, Brussels, Belgium, 2018.

[6] Jingjing Chen and Chong-wah Ngo. Deep-based Ingredient Recognition for Cooking Recipe Retrieval. In Proceedings of the 2016 ACM on Multimedia Conference - MM '16, pages 32-41. ACM Press.

[7] Mei Chen, Kapil Dhingra, Wen Wu, Lei Yang, Rahul Sukthankar, and Jie Yang. Pfid: Pittsburgh fast-food image dataset. In 2009 16th IEEE International Conference on Image Processing (ICIP), pages 289-292. IEEE, 2009.

[8] Manal Chokr and Shady Elbassuoni. Calories Prediction from Food Images. In Proceedings of the Thirty-First AAAI Conference on Artificial Intelligence, AAAI'17, pages 4664-4669, San Francisco, California, USA, 2017. AAAI Press.

[9] Takumi Ege and Keiji Yanai. Image-Based Food Calorie Estimation Using Knowledge on Food Categories, Ingredients and Cooking Directions. In Proceedings of the on Thematic Workshops of ACM Multimedia 2017, Thematic Workshops '17, pages 367-375, New York, NY, USA 2017. ACM.

[10] Hamid Hassannejad, Guido Matrella, Paolo Ciampolini, Ilaria De Munari, Monica Mordonini, and Stefano Cagnoni. Food Image Recognition Using Very Deep Convolutional Networks. In Proceedings of the 2 nd International Workshop on Multimedia Assisted Dietary Management, MADiMa '16, pages 41-49. Association for Computing Machinery.

[11] K. He, X. Zhang, S. Ren, and J. Sun. Deep Residual Learning for Image Recognition. In 2016 IEEE Conference on Computer Vision and Pattern Recognition (CVPR), pages 770-778, June 2016.

[12] Kaiming He, Zhuowen Tu, Piotr Dollár, Ross Girshick, and Saining Xie. Aggregated Residual Transformations for Deep Neural Networks. November 2016

[13] G. Huang, Z. Liu, L. v d Maaten, and K. Q. Weinberger. Densely Connected Convolutional Networks. In 2017 IEEE Conference on Computer Vision and Pattern Recognition (CVPR), pages 2261-2269, July 2017.

[14] 2017 Azumio Inc. Calorie Mama Food AI - Food Image Recognition and Calorie Counter using Deep Learning. https://caloriemama.ai/, [Accessed: 2019-08-17].

[15] Jiatong Li, Ricardo Guerrero, and Vladimir Pavlovic. Deep Cooking: Predicting Relative Food Ingredient Amounts from Images. In Proceedings of the 5th International Workshop on Multimedia Assisted Dietary Management, MADiMa '19, pages 2-6. Association for Computing Machinery.
[16] Yanchao Liang and Jianhua Li. Computer vision-based food calorie estimation: dataset, method, and experiment. arXiv preprint arXiv:1705.07632, 2017.

[17] Javier Marin, Aritro Biswas, Ferda Ofli, Nicholas Hynes, Amaia Salvador, Yusuf Aytar, Ingmar Weber, and Antonio Torralba. Recipe1M+: A Dataset for Learning Cross-Modal Embeddings for Cooking Recipes and Food Images. pages 1-1.

[18] Tomas Mikolov, Ilya Sutskever, Kai Chen, Greg S Corrado, and Jeff Dean. Distributed representations of words and phrases and their compositionality. In Advances in neural information processing systems, pages 3111-3119, 2013.

[19] Weiqing Min, Shuqiang Jiang, Linhu Liu, Yong Rui, and Ramesh Jain. A survey on food computing. ACM Computing Surveys (CSUR), 52(5):136, 2019.

[20] A. Myers, N. Johnston, V. Rathod, A. Korattikara, A. Gorban, N. Silberman, S. Guadarrama, G. Papandreou, J. Huang, and K. Murphy. Im2calories: Towards an Automated Mobile Vision Food Diary. In 2015 IEEE International Conference on Computer Vision (ICCV), pages 1233-1241, December 2015.

[21] US Department of Agriculture, Agricultural Research Service, and Nutrient Data Laboratory. USDA Food Composition Databases. https: //ndb.nal.usda.gov/ndb/, [Accessed: 2019-08-04].

[22] Adam Paszke, Sam Gross, Francisco Massa, Adam Lerer, James Bradbury, Gregory Chanan, Trevor Killeen, Zeming Lin, Natalia Gimelshein, Luca Antiga, Alban Desmaison, Andreas Kopf, Edward Yang, Zachary DeVito, Martin Raison, Alykhan Tejani, Sasank Chilamkurthy, Benoit Steiner, Lu Fang, Junjie Bai, and Soumith Chintala. Pytorch: An imperative style, high-performance deep learning library. In H. Wallach, H. Larochelle, A. Beygelzimer, F. d'Alché-Buc, E. Fox, and R. Garnett, editors, Advances in Neural Information Processing Systems 32, pages 8024-8035. Curran Associates, Inc., 2019.

[23] Parisa Pouladzadeh, Shervin Shirmohammadi, and Abdulsalam Yassine. Using graph cut segmentation for food calorie measurement. In 2014 IEEE International Symposium on Medical Measurements and Applications $(\mathrm{MeMeA})$, pages $1-6$. IEEE.

[24] Olga Russakovsky, Jia Deng, Hao Su, Jonathan Krause, Sanjeev Satheesh, Sean Ma, Zhiheng Huang, Andrej Karpathy, Aditya Khosla, Michael Bernstein, Alexander C. Berg, and Li Fei-Fei. ImageNet Large Scale Visual Recognition Challenge. International Journal of Computer Vision (IJCV), 2015.

[25] Sulfayanti F Situju, Hironori Takimoto, Suzuka Sato, Hitoshi Yamauchi, Akihiro Kanagawa, and Armin Lawi. Food constituent estimation for lifestyle disease prevention by multi-task cnn. Applied Artificial Intelligence, 33(8):732-746, 2019.

[26] Mohammed Ahmed Subhi, Sawal Hamid Ali, and Mohammed Abulameer Mohammed. Vision-based approaches for automatic food recognition and dietary assessment: A survey. IEEE Access, 7:35370 35381, 2019.

[27] Keiji Yanai and Yoshiyuki Kawano. Food image recognition using deep convolutional network with pre-training and fine-tuning. In 2015 IEEE International Conference on Multimedia \& Expo Workshops (ICMEW), pages 1-6. IEEE.

[28] Jun Zhou, Dane Bell, Sabrina Nusrat, Melanie Hingle, Mihai Surdeanu, and Stephen Kobourov. Calorie estimation from pictures of food: Crowdsourcing study. Interactive journal of medical research, 7(2): 17 , 2018.

[29] Fengqing Zhu, Marc Bosch, Carol J. Boushey, and Edward J. Delp. An immage analysis system for dietary assessment and evaluation. pages $1853-1856$. 